\title{
El efecto del cambio climático en especies de plantas vegetales en el altiplano cundiboyacense
}

\author{
The effects of climate change in high andean zones of altiplano cundiboyacense \\ Manuel Torres Torres ${ }^{1}$, Manuel Galvis Rueda ${ }^{2}$ \\ ${ }^{1}$ Candidato a Doctor en Ingeniería Ambiental. Docente Escuela de Ciencias Agrarias, Pecuarias y del \\ Medio Ambiente, Universidad Nacional Abierta y a Distancia - UNAD. Grupo de Investigación \\ GIGASS-Semillero de Investigación Sembrando Nuevas Ideas Agroecológicas. \\ ${ }^{2}$ Magister en Educación. Docente Universidad Pedagógica de Colombia. UPTC. Grupo de \\ Investigación GECOS. \\ 1 manuel.torres@unad.edu.co, 22manuelgalvis@gmail.com
}

\begin{abstract}
Resumen
La influencia del cambio climático genera en las especies nativas y exóticas cambios en su fenología y otros cambios como la fructificación y además la adaptación de especies de otros pisos térmicos y ecosistemas. Así se identificaron los sectores de mayores efectos sobre la cobertura, lo que permitió analizar la situación y proponer acciones de adaptación climática parea la población y los recursos naturales. Se manifiesta en el presente artículo el problema que vienen presentando las especies vegetales con alteraciones de tipo fenológicos en de la zona Cundiboyacense por causa del cambio climático.
\end{abstract}

\begin{abstract}
The influence of climate change on native and exotic species changes in its phenology and other changes such as fruiting and also the adaptation of species of other thermal floors and ecosystems. In this way, the sectors with the greatest effects on the coverage were identified, which allowed analyzing the situation and proposing measures of climate adaptation for population and natural resources. The present problem is manifested in the problem presented by plant species with phenological alterations in the Cundiboyacense area due to climate change.

\section{Introducción}

El presente documento presenta de manera analítica y resumida la evidencia del cambio climático y los efectos causados sobre la especie en la zona de paramo en la región Cundiboyacense. Se analiza la relación que existe entre el cambio climático y la biodiversidad en la zona Cundiboyacense, también, se documentan metodologías y resultados de los efectos del cambio climático, que a medida que pasa el tiempo son más severos en los ecosistemas de la zona. La academia debe proponer acciones investigativas y de extensión que conlleven a la resolución a los problemas que están presentando en las especies, con el acompañamiento y participación de las comunidades. El objetivo es analizar los cambios fenológicos y los efectos de cambios climático en las especies vegetal de la zona Cundiboyacense, presentado en los últimos diez (10) años.
\end{abstract}

\section{Los gases de efecto invernadero y el cambio climático}

Los cambios climáticos en la zona de páramo cundiboyacense, se han manifestados por medio de cambios bruscos en el clima, presentándose temperaturas máximas de $25,5^{\circ} \mathrm{C}$, y mínimas de $3^{\circ} \mathrm{C}$, donde el promedio de temperatura es $14^{\circ} \mathrm{C}$.

Nuestro planeta está rodeado de una fina capa que llamamos atmósfera, que actúa a modo de cubierta protectora haciendo que la temperatura en la superficie terrestre sea la idónea para el desarrollo de la vida. La atmósfera está compuesta por una serie de gases entre los que destacan el nitrógeno y el oxígeno, además de otra gama de gases en menores proporciones. 
La retención de esta energía provoca el incremento de la temperatura de la superficie terrestre, siendo la media de unos $15^{\circ} \mathrm{C}$, óptima para el desarrollo de la vida.

Sin este proceso de retención de la radiación, la temperatura en la superficie de la Tierra sería notablemente inferior, del orden de $30^{\circ} \mathrm{C}$ menos, por lo que la vida se haría casi imposible, de tal modo que la presencia de estos gases es imprescindible.

\section{Conceptos relacionados con el cambio climático.}

Clima. Refiere al estado de la atmósfera alrededor de valores promedio, producto de la evaluación de observaciones de largos periodos de tiempo mayores o iguales a 30 años, conocidos como Normales Climatológicas (IDEAM, 2011).

Variabilidad climática Se refiere a las fluctuaciones observadas en el clima, alrededor de una condición promedio, durante periodos de tiempo relativamente cortos. La variación observada en el clima durante periodos consecutivos de varias décadas se llama cambio climático (IDEAM, 2011).

Vulnerabilidad De acuerdo con el IPCC, la vulnerabilidad es el grado de susceptibilidad o de incapacidad de un sistema para afrontar los efectos adversos del cambio climático, incluyendo la variabilidad climática y los fenómenos extremos. La vulnerabilidad dependerá del carácter, la magnitud y la rapidez del cambio climático a que esté expuesto un sistema, y de su sensibilidad y capacidad de adaptación (IDEAM, 2011).

Impactos del cambio climático. A continuación, se presentan algunos impactos proyectados del cambio climático, consignados principalmente en el documento resumen del Grupo I del IPCC para el Cuarto Reporte de Evaluación (IPCC, 2007):

- Aumento en el promedio de la temperatura superficial global en un rango entre $2{ }^{\circ} \mathrm{C}$ y $4,5{ }^{\circ} \mathrm{C}$ cuando se presente una duplicación en las concentraciones de $\mathrm{CO}_{2}$ respecto al valor preindustrial (280 partes por millón [ppm]), el cual se podría alcanzar a finales de este siglo.

Sequias

Los investigadores están pronosticando con seguridad de que el Amazonas, el noreste de Brasil, Centro América y partes de México sufrirán mayores sequías. La principal preocupación es la posibilidad de sequías extremas y más frecuentes en el Amazonas, lo cual podría orillar a la región hasta un "punto de no retorno", lo que aumentaría la probabilidad de que se perdieran grandes cantidades de selva amazónica.

Entre las sequías recientes que afectaron el Amazonas destacan las de 2005 y 2010. Asimismo, las actuales condiciones de sequía en México, Centro América y el Caribe pueden intensificarse debido al fuerte fenómeno de El Niño 2015-2016, que está ocurriendo en un contexto de temperaturas más altas asociadas con el calentamiento global.

\section{Aumento del nivel del mar y acidificación del océano}

Gran parte del área costera que rodea al arrecife mesoamericano e islas cercanas son zonas bajas y por lo tanto vulnerables al aumento del nivel del mar provocado por el cambio climático. La erosión costera ya ha sido documentada y, entre otras cosas, puede afectar los procesos de anidación y reproducción de las tortugas marinas. El aumento de la temperatura del agua genera más episodios de blanqueamiento de los corales, lo cual es devastador para los arrecifes y toda la vida marina que depende de ellos. La acidificación del océano, provocada por el aumento de la concentración de dióxido de carbono en el agua, es otra amenaza que afecta a los arrecifes coralinos.

\section{Fenómenos meteorológicos extremos}

Los cambios en el clima y los fenómenos meteorológicos extremos han afectado severamente a América Latina. Eventos como tifones y huracanes, tormentas eléctricas, granizadas fuertes nevadas, aludes, inundaciones incluyendo inundaciones repentinas, sequías, olas de calor y olas de frío, heladas son cada vez más frecuentes y severos. Todo esto ha provocado el desplazamiento de 
personas, numerosas muertes, afectación en la producción agropecuaria e importantes pérdidas económicas.

\section{Materiales y métodos}

Los métodos que se aplicaron fueron una investigación de enfoque mixto, porque representa la integración sistemática del método cuantitativo y cualitativo (Hernández et al., 2010) con el fin de que la información que se obtuvo sea más significativa, utilizando un alcance descriptivo exploratorio -transversal, así mismo. Se procedió a realizar observaciones y registros en campo, analizando los fenómenos que se presentan a través del tiempo, luego se realizó una identificación de las colecciones de herbario: se revisaron especímenes depositados en el herbario (UPTC y COL), se estudió la información bibliográfica relacionada con la taxonomía, descripciones y listados de floras regionales, igual análisis de fuentes secundarias.

\section{Evidencia de cambio climático en Colombia}

Se ha considerado que el cambio climático traerá efectos negativos en nuestro país en los diferentes componentes de la vida actual (PNUD, 2009b). Entre estos:

- Aumento de la temperatura entre 2 y $4{ }^{\circ} \mathrm{C}$, especialmente a lo largo de la región andina y en el Caribe.

- Para las siguientes dos décadas se proyecta un calentamiento de cerca de $0,2^{\circ} \mathrm{C}$ para el rango de escenarios de emisión. Incluso si las concentraciones de todos los GEI y aerosoles se mantuvieran constantes a los niveles del año 2000 , se esperaría un calentamiento adicional de cerca de $0,1^{\circ} \mathrm{C}$ por década (IDEAM, 2011).

- Cambios importantes en el régimen hidrológico (ciclo del agua), con una reducción de las lluvias de hasta $30 \%$, principalmente en la región andina, lo cual incrementará el déficit hídrico de algunas cuencas abastecedoras de acueductos, distritos de riego y embalses hidroeléctricos.

- Aumento de la recurrencia e intensidad de eventos hidrometeorológicos extremos, como inundaciones, deslizamientos, vendavales, avenidas torrenciales, granizadas, temporadas de calor en zonas altas y sequías; se prevé un incremento de la cantidad de personas afectadas.

- Desplazamiento de ecosistemas naturales hacia zonas de vida más secas y especial afectación y disminución del área de ecosistemas estratégicos, como los páramos y nevados $(75 \%$ de los páramos y $95 \%$ de los nevados podrían desaparecer).

- Disminución y afectación de los bienes y servicios ambientales de todos los ecosistemas, con la consecuencia del aumento de los conflictos por acceso y uso de los recursos naturales.

Con respecto a las evidencias actuales, se destacan estudios que analizan la información hidroclimática (Pérez, Pobeda, Mesa, Carvajal \& Ochoa, 1998) y señalan una variación en caudales de los ríos Magdalena y Cauca, así como de temperatura, punto de rocío y temperatura en algunas regiones del país, que serían una posible variación por efecto del cambio climático.

Colombia, aunque sólo contribuye con el $0,37 \%$ de las emisiones de Gases de Efecto InvernaderoGEl (2004), es vulnerable al cambio climático.

\section{Fragilidad de los ecosistemas de páramo}

Los suelos de los ecosistemas de páramo, tienen un bajo nivel de resiliencia por lo que son considerados muy frágiles. Alrededor de $3 / 4$ partes de los suelos en el páramo corresponden a agua. En las zonas de Páramo en el departamento de Boyacá, los campesinos a través del tiempo han venido ampliando las fronteras agrícolas para obtener ganancia en sus cultivos, dentro de estos procesos productivos, el pastoreo tiene efectos profundos en la estructura y funcionamiento de los páramos. El pisoteo del ganado genera compactación del suelo y pérdida de las propiedades retenedoras de agua y carbono. Sus efectos son notorios en la relación entre la distribución de la biomasa que se encuentra por encima del suelo (epigea) y la biomasa que está por debajo del suelo (hipogea), la fisionomía de las plantas y la ocurrencia del mismo fuego. No obstante, a pesar de la 
gran influencia humana en los páramos, la cobertura en general es dominada por flora nativa. Existen enclaves de tipo azonal, como comunidades que se encuentran en suelos saturados de aguas, turberas y lagunas (IDEAM, Ministerio del Medio Ambiente y Programa de las Naciones Unidas para el Desarrollo, 2002). Los paisajes conservados de los ecosistemas de páramo son aquellos que presentan un bajo régimen de disturbios de origen antrópico. Como los suelos contienen alrededor de tres veces más reservas de carbono orgánico que la biomasa vegetal y animal sobre la tierra y el doble del carbono contenido en la atmósfera, la materia orgánica del suelo es potencialmente la fuente más peligrosa de $\mathrm{CO} 2$ para el calentamiento global, aparte del causado por la quema de combustibles fósiles (carbón, petróleo y gas). Indirectamente, el descenso en la producción agrícola y de biomasa resultante de la degradación de los suelos también contribuye a un incremento del CO2 atmosférico, por descenso en el secuestro de $\mathrm{CO} 2$ en la fotosíntesis y por obligar a incorporar nuevas tierras a la producción agrícola, generalmente a través de deforestaciones incontroladas y quemando la vegetación, especialmente en zonas ecuatoriales.

Según (Luteyn 1992), citado por (Maya \& Bolaños 2011) declara que "Los páramos tienen un importante valor científico y ecológico por su flora endémica y su paisaje, desempeñan una función en la producción de alimentos y regulan la hidrología regional como constituyente de la fuente de agua potable para la mayoría de la población de la parte alta de los Andes". Por esta razón es de gran importancia buscar estrategias de mitigación del cambio climático en la zona

\section{Los efectos del cambio climático sobre los ecosistemas de páramo}

Los resultados de estudios realizados por el IDEAM indican que en un escenario de duplicación de $\mathrm{CO} 2$ en la atmósfera, la temperatura aumentará entre 2.5 y $3^{\circ} \mathrm{C}$, reduciéndose la precipitación entre un 10 y $20 \%$. El principal efecto potencial es el probable ascenso de las zonas bioclimáticas y sus límites hasta unos 400 a 500 metros, en un tiempo relativamente corto. Estas partes de las zonas de vida bioclimáticas según Holdridge 3 que sufrirían la transición a otra zona serían las más vulnerables a los impactos del cambio climático. Es importante resaltar que estamos frente a la proximidad de una situación que no se ha presentado en por lo menos 700 mil años o más (IDEAM, Ministerio del Medio Ambiente y Programa de las Naciones Unidas para el Desarrollo, 2002).

Por otro lado, según un estudio de la Food administration organization FAO, entre los años 2003 y 2013, las inundaciones, sequías y otros desastres causaron el $83 \%$ de las pérdidas de cultivos y ganado en los países en desarrollo. En América Latina, las pérdidas en el sector agrícola durante ese periodo fueron de 11.000 millones de dólares, en su mayoría por los desastres asociados al clima.

Según Ocampo (2011) Existen evidencias científicas sobre el impacto del cambio climático en la agricultura por el efecto sobre las variables conexas relevantes: la temperatura, la precipitación, la concentración de dióxido de carbono y la humedad del suelo. Sin embargo, las prácticas de gestión, los cambios tecnológicos, la dinámica del mercado y las políticas públicas afectan el sector y crean incertidumbres, por la dificultad de evaluar de forma aislada la influencia del clima, con respecto a los otros factores

Según FAO (2001) El cambio climático también será responsable por el aumento en la frecuencia y severidad de los incendios forestales. Los incendios no solo serán más intensos y frecuentes, sino que, además, se extenderán hacia ecosistemas que tradicionalmente no se incendiaban, afectando la biodiversidad del entorno.

Según Uribe Botero, E. (2015). La pérdida de especies y el deterioro de los ecosistemas pueden afectar los flujos de bienes y servicios que los ecosistemas proporcionan a la sociedad. Así, por ejemplo, la disminución de la cubertura boscosa puede llegar a generar una reducción de la precipitación a escala regional y local.

Por otro lado, Según Galvis y Torres M (2017) el conocer los sitios y condiciones

en donde las plantas existen, como se propagan, cuando se cosechan y cuando se siembran son algunos de los factores que nos permiten entender los requerimientos de propagación de las plantas. Este conocimiento facilita prácticas para recuperar plantas que estén en peligro de extinción, y es una estrategia que se puede utilizar para mitigar el cambio climático. 
Pero hay otras estrategias para poder mitigar el efecto de cambio climático con acciones de implementación de la agroecología y reducción de la aplicación de agrotoxicos, por parte de la comunidad, como lo refiere Mendoza, Cano y Rojas 2015 en su Investigación Acción Participante que utilizaron en la Evaluación de Sistemas de Manejo incorporando Indicadores de Sustentabilidad (MESMIS) y la cartografía social para el diagnóstico. Se realizaron sesiones de reflexión y aprendizaje con la comunidad a partir de los resultados.

\section{Resultados y discusión}

En el contexto de la zona cundiboyacense se presenta un fenómeno influenciado por el cambio climático; se observa un cambio en la fenología de las especies que son generada por las variaciones en la precipitación y en la temperatura, que inducen a cambios en los ecosistemas en región, esto trae como consecuencia la reducción de plantas nativas como mortiño Hesperomeles goudotiana (Decne.) Killip hayuelo, Dodonaea viscosa (L.) Jacq., Aliso Alnus glutinosa Kunth, roble Quercus (humboldtii) Bonpl., y Cerezo Prunus serótina var. Capulí (Cav.) McVaugh. (Bartholomäus A. 1990). Un ejemplo de estos cambios fenológicos se presenta en la floración de los robles, con bellotas más pequeñas, y muchas de estas son infértiles.

Otro ejemplo de cambios fenológicos es en el árbol del raque Vallea stipularis .L en el color de las hojas (rojo escarlata), es más intenso y con el tiempo la planta muere por estrés hídrico.

En el Mangle Escallonia péndula se presenta caídas de hojas produciendo la muerte descendente de la especie.

En relación con el aumento de la temperatura y la fragmentación del ecosistema se ha observado el desarrollo de epifitas como Tillandsia recurvata L. Bromeliacea, sobre troncos de árboles principalmente en el dividivi Caesalpinia spinosa (Molina) Kuntze., que saturan los tallos y causan la muerte del hospedero.

Otro fenómeno se presenta en el páramo de la Rusia cerca la ciudad de Duitama (Boyacá), hay aumento de macrofita, en los cuerpos de aguas, especies exótica invasoras como Typha domingensis. Pers y Schoenoplectus californicus (C.A. Mey.) Soják, causando en el sitio perdida del espejo de agua y acelerado el proceso de eutrificación.

En áreas de cultivos la invasión de Rumex acetosella L. Hierba introducida del África., propia en los estratos bajos de robledal degradado y en áreas de cultivo, presentando saturación y acarreado costos adicionales en cultivos propio de la región como la papa (Solanum tuberosum) .L.

Por otra parte, se presentó el cambio en la fenología del aguacate (Persea americana) L. La distribución hubo floración y fructificación en el año 2016, en una altura de 2780 m.s.n.m. (Ubicación Tunja Boyacá, Predios de la Universidad Pedagógica y Tecnológica de Colombia) en zona de paramo. En otros sitios como la ciudad de Sogamoso a una altura de 2.475 m.s.n.m. y la ciudad de Duitama a 2530 m.s.n.m., con temperaturas promedios de $15^{\circ} \mathrm{C}$ con una aclaración estos árboles de aguacate tienen más de 15 años.

Según J.J Pérez el aguacate (Persea americana. L), el medio ambiente óptimo para su desarrollo es de 0 a 1.000 m.s.n.m. y temperatura media de $29^{\circ} \mathrm{C}$., pero en Tunja situada a 2.800 m.s.n.m. y Duitama situada a 2.530 m.s.n.m. se está presentando floración y fructificación en la planta.

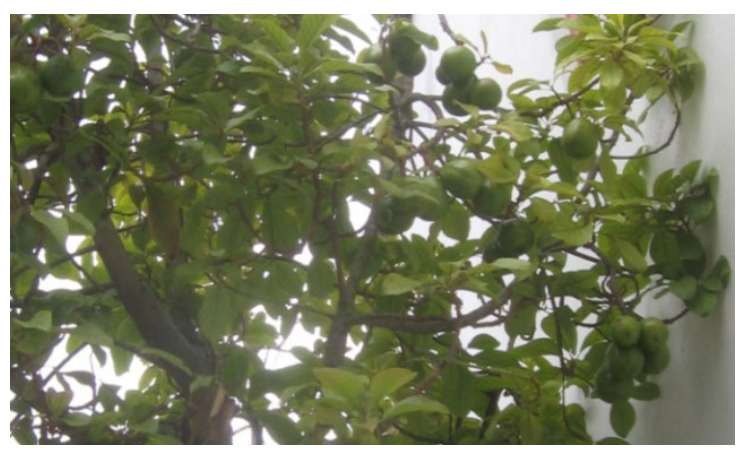


Figura 1. Fructificación de Aguacate (Persea americana) en la ciudad de Tunja a una altura de 2780 m.s.n.m. Fuente: Autores

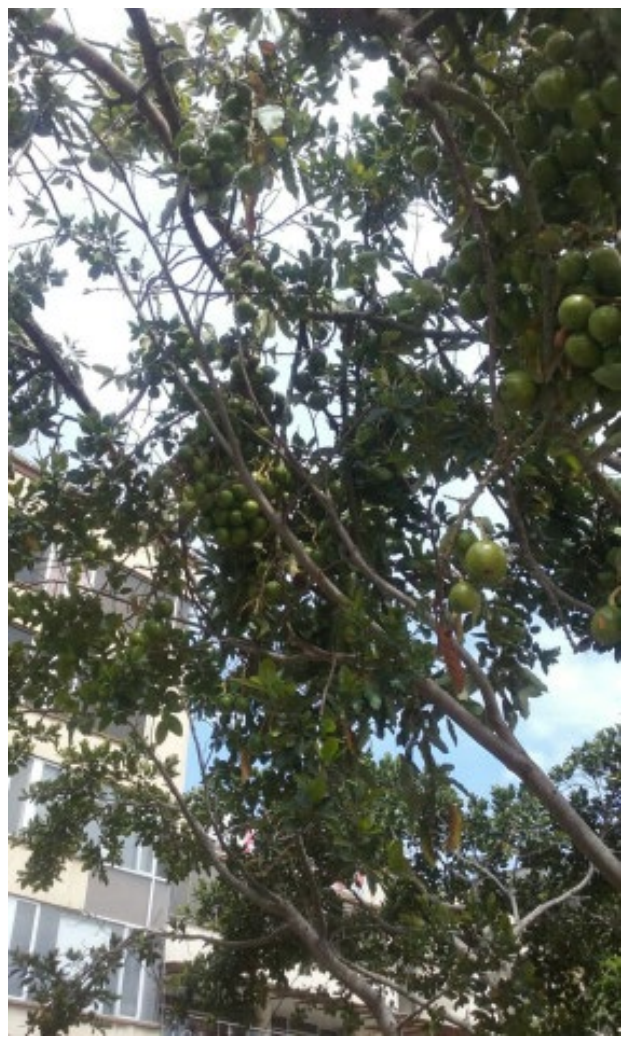

Figura 2. Fructificación de Aguacate (Persea americana) en la ciudad de Duitama a una altura de 2530 m.s.n.m. Fuente: Autores

De los factores climáticos que causan graves consecuencias en las especies se encuentran las heladas, fenómeno que a través de los años se ha venido incrementando en la zona Cundiboyancense.

Se relacionan los incendios ocurridos en los últimos tres años en el departamento de Boyacá afectando a más de 60 municipios de la totalidad 123, causado por el fenómeno del cambio climático En la tabla No 1 se presenta un listado de especies y los daños causados por el fenómeno del cambio climático en ellas.

\begin{tabular}{|c|c|c|c|c|c|}
\hline $\begin{array}{l}\text { Nombre } \\
\text { común }\end{array}$ & Familia & Genero & Especies & Efectos & Distribución Geográfica/origen \\
\hline Mortiño & Rosaceae & Hesperomeles & $\begin{array}{l}\text { goudotiana (Decnec) } \\
\text { Killip }\end{array}$ & Muerte & $\begin{array}{l}\text { Andina, Colombia. Árbol nativo. Crece en } \\
\text { matorrales densos (procesos de sucesión } \\
\text { vegetal de siete años), áreas de páramo y } \\
\text { bosques relictuales. }\end{array}$ \\
\hline Hayuelo & Sapindáceas & Dodonaea & viscosa (L.) Jacq. & Muerte & $\begin{array}{l}\text { Andina, Colombia. Arbusto nativo. Crece en } \\
\text { áreas de procesos sucesionales de quince } \\
\text { años de edad, matorrales densos con } \\
\text { dominancia. }\end{array}$ \\
\hline Aliso & Betulaceae & Alnus & acuminata Kunth & $\begin{array}{l}\text { Muerte descendente, } \\
\text { ataque de plaga }\end{array}$ & $\begin{array}{l}\text { Andina, Colombia. Árbol nativo. Frecuente al } \\
\text { borde de carreteras y en áreas cercanas a } \\
\text { cultivos y potreros }\end{array}$ \\
\hline Roble & Fagaceae & Quercus & humboldtii Bonpl. & $\begin{array}{l}\text { Muerte y fructificación } \\
\text { vana }\end{array}$ & $\begin{array}{l}\text { Andina, Colombia. Árbol nativo. Crece en } \\
\text { bosques, forma asociaciones homogéneas. }\end{array}$ \\
\hline
\end{tabular}




\begin{tabular}{|c|c|c|c|c|}
\hline Cerezo & Rosaceae & Prunus & $\begin{array}{l}\text { serotina var. capuli } \\
\text { (Cav.) McVaugh }\end{array}$ & Muerte \\
\hline Fique & $\begin{array}{l}\text { Agavaceae- } \\
\text { Amaryllidaceae }\end{array}$ & Furcraea & cabuya Trel & Floración muerte \\
\hline Raque & $\begin{array}{l}\text { Elaeocarpacea } \\
\text { e }\end{array}$ & Valled & stipularis L.f & Muerte \\
\hline Barba & Bromelaceae & Tillandsia & recurvata (L.) L. & $\begin{array}{l}\text { Proliferación } \\
\text { fructificación }\end{array}$ \\
\hline Dividivi & caesalpinaceae & Caesalpinia & $\begin{array}{l}\text { spinosa } \\
\text { Kuntze }\end{array}$ & Muerte total \\
\hline Cipres & Cupressaceae & Cupressus & lucitannica Mill & Muerte total \\
\hline Chusque & Poaceae & Chusquea & scandens Kunth. & $\begin{array}{l}\text { Floración, } \\
\text { foliar }\end{array}$ \\
\hline kikuyo & Poaceae & Pennisetum & $\begin{array}{l}\text { clandestinum } \\
\text { Schumach. }\end{array}$ & Muerte foliar \\
\hline Helecho & Blechnaceae & Blechnum & occidentale L. & Muerte de fronde \\
\hline Arrayan & Myrtaceae & Myrcianthes & $\begin{array}{l}\text { leucoxyla } \\
\text { Mc.Vaugh }\end{array}$ & Muerte total \\
\hline
\end{tabular}

Andina, Colombia. Árbol nativo. Crece al borde de caminos, en áreas de cultivo

México. Arbusto Exótico. Roseta frecuente en suelos degradados, con erosión avanzada, al borde de carretera y cultivada.

Andina, Colombia. Árbol nativo. Frecuente al borde de carreteras, en zonas de bosque relictual y páramo. Vereda Torres y Valero, en la Reserva

Andina, Colombia. Roseta epifita, nativa. Crece sobre el árbol de Cesalpinácea. Frecuente, en áreas con alto grado de degradación de suelos Andina, Colombia. Árbol nativo. Crece en áreas de matorrales, potreros, bosques recuperados y bordes de camino.

Exótica USA. Árbol introducido y cultivado.

Andina, Colombia. Arbusto nativo. Crece en áreas de subpáramo. Vereda Firita Peña Arriba, $3050 \mathrm{~m}$

Exótica-África. Hierba introducida al país Crece en áreas húmedas y desérticas, zonas de bosque y vegetación de estratos herbáceos Andina, Colombia. Hierba nativa, que crece en los bordes de la quebrada.

Andina, Colombia. Árbol nativo. Crece en matorrales y en vegetación de subpáramo, sobre suelos secos con erosión moderada, Colombia

\begin{tabular}{|c|c|c|c|c|c|}
\hline $\begin{array}{l}{ }^{*} \text { Acacia } \\
\text { blanca }\end{array}$ & Mimosacea & Acacia & dealbata Link & Muerte & $\begin{array}{l}\text { Exótica- Australia. Acacia dealbata, la mimosa, } \\
\text { acacia mimosa, aromo o acacia australiana es } \\
\text { una especie arbustiva o arbórea perteneciente } \\
\text { a la familia de las leguminosas. }\end{array}$ \\
\hline Junca & Cyperaceae & Schoenoplectus & $\begin{array}{l}\text { californicus (C.A. } \\
\text { Mey.) Soják }\end{array}$ & Proliferación & $\begin{array}{l}\text { Exótica-USA. Planta de agua que se encuentra } \\
\text { en las zonas pantanosas. Es nativa del sur y el } \\
\text { oeste Estados unidos, tallo es alto, delgado, de } \\
\text { color verde oscuro. }\end{array}$ \\
\hline Junco & Juncaceae & Juncus & effusus $L$. & Proliferación & $\begin{array}{l}\text { Es una planta perenne herbácea, casi Effusus } \\
\text { juncus crece en grandes grupos alrededor de } \\
1,5 \text { metros ( } 4 \text { pies } 11 \text { pulg) de alto en la orilla } \\
\text { del agua a lo largo de los arroyos y zanjas, pero } \\
\text { puede ser invasiva en cualquier lugar con el } \\
\text { suelo húmedo. Se encuentra comúnmente } \\
\text { crece en zonas ricas en humus como } \\
\text { pantanos, acequias, pantanos y diques }\end{array}$ \\
\hline Enea & Thiphaceae & Thipha & domingues $\mathrm{L}$. & Proliferación & $\begin{array}{l}\text { Hierba Exótica y acuática, en áreas inundadas, } \\
\text { formando grandes grupos. Crece en áreas de } \\
\text { regeneración no intervenida hace quince años }\end{array}$ \\
\hline
\end{tabular}

En la tabla No 2 de agrupamiento de afectación por el cambio climático, se observa que la muerte de árboles y arbustos es la frecuente, seguida de la defoliación de las especies, a pesar de que algunas tengan estrategias evolutivas de supervivencia.

Tabla 2. Datos de las afectaciones a las especies

Efectos

Muerte

Defoliación
No

12

4

Vol. 2, Núm. 1 (2018) 


$\begin{array}{ll}\text { Ataque plagas } & 2 \\ \text { Floración } & 2 \\ \text { Cambio de coloración } & 2 \\ \text { Proliferación } & 3 \\ \text { Cambio fenológico } & 1\end{array}$

Fuente: Autores

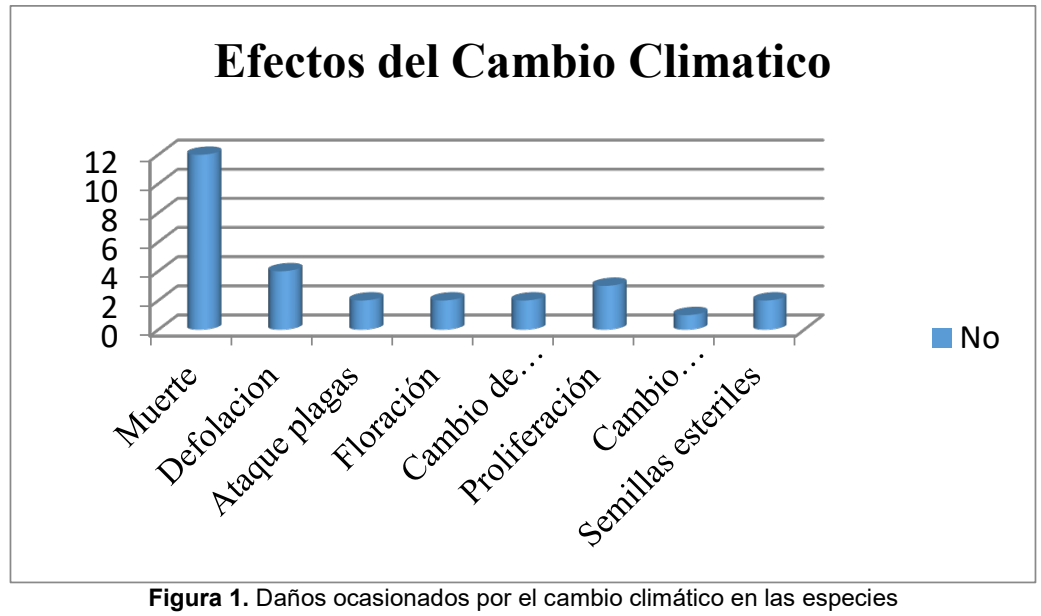

Fuente: Autores

Como se observa en la figura 1 son variados los efectos en la compleja diversidad de especies de la zona de páramo, lo que conlleva a una reflexión que a medida que pasa el tiempo se incrementa el desequilibrio ambiental y social, trayendo como consecuencia problemas socioeconómicos a la región.

\section{Conclusiones}

El fenómeno del cambio climático está generando daños en las especies vegetales de ecosistemas de clima frio, como muerte foliar, defoliación, cambio de coloración en plantas, cambio fenológico, semillas estériles, y adaptación de especies foráneas de otros pisos térmicos, originando invasión y extinción de algunas especies de la zona, ocasionando desplazamiento de la fauna, ante la pérdida de su habitad, todo esto a causa de variaciones extremas de la temperatura y de falta de precipitaciones.

Se analizó la situación y |se propone acciones por parte de la academia para realizar propuestas de investigación y de extensión, con la participación de la comunidad, para la disminución del impacto del cambio climático en las especies nativas de la región Cundiboyacense.

\section{Literatura citada}

Bartholomäus A. (Ed.). (1990). El Manto de la Tierra: Flora de Los Andes: Guía de 150 Especies de la Flora Andina. Bogotá, Colombia: Corporación Autónoma Regional de las Cuencas de los Ríos Bogotá, Ubaté y Suárez.

FAO - Organización de las Naciones Unidas para la agricultura y la alimentación. (2001). Agricultura y Cambio Climático. Recuperado de: http://www.fao.org/ag/esp/revista/0103sp2.htm 
FAO - Organización de las Naciones Unidas para la agricultura y la alimentación. (2002). Consecuencias del Cambio Climático. Recuperado de: http://www.fao.org/docrep/017/i2498s/i2498s04.pdf

Galvis R. Manuel, Torres T. Manuel. (2017). Etnobotánica y usos de las plantas de la comunidad rural de Sogamoso, Boyacá, Colombia. Revista de Investigación Agraria y Ambiental, 8(2), 187- 206. https://doi.org/10.22490/21456453.2045

Hernández, R., Collado, F. \& Baptista, P. (2010). Metodología de la investigación. (5th ed.) México: McGrawHill.

IDEAM - Instituto de Hidrología, Meteorología y Estudios Ambientales. (2010). 2a Comunicación Nacional ante la Convención Marco de las Naciones Unidas sobre el Cambio Climático. Recuperado de: http://bit.ly/2tvzuBk

IDEAM - Instituto de Hidrología, Meteorología y Estudios Ambientales. (2011). Evidencias de cambio climático en Colombia con base en información estadística. Recuperado de: http:// http://www.ideam.gov.co/documents/21021/21138/

IDEAM - Instituto de Hidrología, Meteorología y Estudios Ambientales. (2002). Libro de páramos y Ecosistemas Alto Andino de Colombia. Recuperado de: http://bit.ly/2tZGisW

IPCC. (2007). Cambio climático 2007: Informe de síntesis. Recuperado de: https://www.ipcc.ch/pdf/assessment-report/ar4/syr/ar4_syr_sp.pdf

Mahecha, G., Ovalle, A., Camelo, D., Rozo, A., \& Barrero, D. (2004). Vegetación del territorio CAR: 450 especies de sus llanuras y montañas. Bogotá: Corporación Autónoma Regional de Cundinamarca.

Maya Pantoja, J., \& Bolaños Lopez, A. (2011). Conservación y uso sostenible en la zona de amortiguamiento del páramo de Chiles, departamento de Nariño. Revista de Investigación Agraria y Ambiental, 2(1), 75-82. doi:https://doi.org/10.22490/21456453.907

Mendoza Velásquez, S., Cano Muñoz, J., \& Rojas Sánchez, F. (2015). Acción comunitaria frente al fenómeno del cambio climático, en el páramo de la región del Guavio, Cundinamarca, Colombia. Revista de Investigación Agraria y Ambiental, 6(1), 265 - 279. doi:https://doi.org/10.22490/21456453.1286

Ocampo, O. (2011) El cambio climático y su impacto en el agro. Revista de ingeniería 33, 115-123.

PDUN (2009) Cambio climático Ciencia, evidencia y acciones. México.

Pérez, J.J. (2000). Cultivo II. Bogotá, Colombia: Universidad Abierta y a Distancia.

Pérez, C., \& Poveda, G., \& Mesa, O., \& Carvajal, L., \& Ochoa, A. (1998). Evidencias de cambio climático en Colombia: tendencias y cambios de fase y amplitud de los ciclos anual y semianual. Bulletin de I'Institut français d'études andines, 27 (3).

Uribe Botero, E. (2015). El cambio climático y sus efectos en la Biodiversidad. Editorial Cepal. 